CAE Working Paper \#03-05

Utilitarianism for Infinite Utility Streams:

A New Welfare Criterion and its Axiomatic

Characterization

by

Kaushik Basu

\&

Tapan Mitra

May 2003 


\title{
Utilitarianism for Infinite Utility Streams: A New Welfare Criterion and its Axiomatic Characterization*
}

\author{
Kaushik Basu ${ }^{\dagger}$ and Tapan Mitra ${ }^{\ddagger}$
}

May 20, 2003

\begin{abstract}
A definition of a utilitarian social welfare relation (SWR) for infinite utility streams is proposed. Such a relation is characterized in terms of the Pareto, Anonymity and Partial Unit Comparability Axioms. The merits of the utilitarian SWR, relative to the more restrictive SWR induced by the overtaking criterion, are examined.

Journal of Economic Literature Classification Numbers: D60, D70, D90.

Key Words: intergenerational equity, Pareto, grading principle, social welfare relations, utilitarianism, partial unit comparability, overtaking criterion.
\end{abstract}

\footnotetext{
*The authors are grateful to Jorgen Weibull for helpful suggestions.

${ }^{\dagger}$ Department of Economics, Cornell University, Ithaca, NY 14853, Email: kb40@cornell.edu.

${ }^{\ddagger}$ Department of Economics, Cornell University, Ithaca, NY 14853, Email: tm19@cornell.edu.
} 


\section{Introduction}

In comparing infinite utility streams, two guiding principles have generally been found to be widely acceptable. If, like Ramsey (1928), we would like to treat all generations equally, we have to accept the Anonymity Axiom. ${ }^{1}$ If our intertemporal preference structure is to be (positively) sensitive to the well-being of each generation, we are led to impose the Pareto Axiom. ${ }^{2}$ It would be convenient if one could construct a social welfare function (SWF) which respected both of these principles, because then comparisons of infinite utility streams could be conveniently carried out in terms of the social welfare numbers associated with the utility streams.

In fact, it would be futile to try to construct such a social welfare function, because it can be shown that that there is no social welfare function which respects both the Anonymity and the Pareto axioms. In other words, all Paretian social welfare functions are necessarily inequitable. ${ }^{3}$

This need not deter progress however, because if one could construct a social welfare ordering (SWO) respecting the two axioms, we would be able to compare all infinite utility streams in terms of this ordering. Svensson (1980) was the first to show that such an ordering does exist. However, it is worth noting that he obtains the ordering by non-constructive methods; specifically, he defines a pre-order (a binary relation satisfying reflexivity and transitivity) satisfying the two axioms, and then completes the order by appealing to Szpilrajn's lemma. ${ }^{4}$ Thus, knowing that such an ordering exists

\footnotetext{
${ }^{1}$ Many authors have felt that a stronger notion than the Anonymity Axiom is needed to reflect intergenerational equity in intertemporal preferences. However, there appears to be general agreement that any notion of intergenerational equity in intertemporal preferences must include the Anonymity Axiom.

${ }^{2}$ The Rawlsian Social welfare function, which figures quite prominently in discussions on equity, violates the Pareto principle, even in comparisons of utility streams where each utility stream has a well-defined minimum. For example, in comparing $x$ and $x^{\prime}$, where $x_{1}=x_{1}^{\prime}=0.4$, and $x_{n}=0.5+(1 / n)$ for $n \geq 2, x_{n}^{\prime}=0.5$ for $n \geq 2, x$ is clearly Paretosuperior to $x^{\prime}$, but since $\min _{n \geq 1} x_{n}=0.4=\min _{n \geq 1} x_{n}^{\prime}$, the Rawlsian SWF would consider the utility sequences to be indifferent.

${ }^{3}$ While this impossibility result might sound familiar, it has actually been established only recently in Basu and Mitra (2002), without any domain restriction and without any other axiom imposed on preferences. The well-known impossibility result of Diamond (1965) was established for a specific domain and, more importantly, with an additional continuity axiom on preferences.

${ }^{4}$ Recall that a standard way of proving Szpilrajn's Lemma is by using Zorn's Lemma, which is known to be equivalent to the Axiom of Choice. See, for example, Fishburn
} 
does not necessarily provide a clue as to how it might be constructed.

In view of this, we might consider lowering our demands further and be willing to accept social welfare relations (SWR) which are pre-orders that allow (consistent) comparisons between only some pairs of infinite utility streams but not others. ${ }^{5}$ In this case, one can actually construct several social welfare relations satisfying the Anonymity and Pareto Axioms. The SuppesSen grading principle ${ }^{6}$ and the pre-orders induced by the "overtaking" or "catching-up" criterion ${ }^{7}$ are examples of such social welfare relations.

One way to be selective among such SWRs is to impose an axiom ensuring some degree of intertemporal comparability of utilities. In the context of intertemporal preferences, a partial (cardinal) unit comparability axiom appears to be a natual comparability requirement to have. If we do so, we obtain an interesting social welfare relation which compares only those infinite utility streams which are "Pareto comparable" beyond a finite horizon, and which applies standard utilitarian principles up to that finite horizon.

This utilitarian social welfare relation satisfies the Anonymity, Pareto and Partial Unit Comparability axioms. It turns out that it is the least restrictive pre-order which does so. ${ }^{8}$ If any SWR satisfies the three axioms, then the utilitarian SWR is a subrelation to it in the sense that the rankings of the utilitarian SWR must always be respected by any such SWR. In this sense, the utilitarian social welfare relation is characterized by the Anonymity, Pareto and Partial Unit Comparability axioms. ${ }^{9}$

(1970) for a proof of Szpilrajn's Lemma.

${ }^{5}$ Pre-orders, incomplete though they may be, have turned out to be powerful tools. The use of the Lorenz pre-order in studies of income inequality, and the pre-order induced by the overtaking criterion in optimal growth theory, are two well-known examples.

${ }^{6}$ The Grading Principle is due to Suppes (1966). For a comprehensive analysis of it, see Sen (1971).

${ }^{7}$ For the definitions of the SWRs appropriate to this discussion, see section 4 .

${ }^{8}$ To elaborate, if one identifies a binary relation with its graph, then the set $S_{U}$ representing the graph of the utilitarian SWR is the smallest set (in terms of the partial order of $\subset$ ) among all sets representing graphs of SWRs which satisfy the Anonymity, Pareto and Partial Unit Comparability Axioms. This is discussed fully in Section 3.1.

The SWRs induced by the overtaking criterion (discussed in detail in Section 4) satisfy all three axioms, but they are clearly not the least restrictive SWRs which do so. The Suppes-Sen grading principle is, of course, a less restrictive pre-order than the utilitarian SWR, but it does not satisfy the Partial Unit Comparability axiom. This point is discussed in Section 3.2 below.

${ }^{9}$ In the same sense, the Suppes-Sen grading principle is characterized by the Anonymity and Pareto Axioms. See the discussion in Section 3.2 below. 
We compare our utilitarian SWR with the SWRs induced by the overtaking or catching-up criteria in Section 4. A noteworthy feature of our utilitarian SWR is that it is axiomatized without postulating any continuity property on the pre-order in the infinite dimensional space containing the set of utility streams. In contrast, axiomatic characterizations of the more restrictive SWRs induced by the overtaking criterion typically involve some form of a continuity axiom. ${ }^{10}$

We argue that the rankings provided by our utilitarian SWR are more widely acceptable than the rankings provided by the overtaking SWR. Of course, the overtaking (and more so the catching-up) SWR provides rankings of two utility streams in many cases in which the utilitarian SWR finds them non-comparable. That is, the utilitarian SWR is more incomplete than the overtaking SWR. But, it turns out that, at least for a class of intertemporal allocation models, this incompleteness is not a handicap in characterizing dynamic optimal behavior, and the power of the overtaking SWR to rank a larger set of utility streams than the utilitarian SWR is found to be quite superfluous.

\section{Notation and Definitions}

Let $\mathbb{N}$ denote, as usual, the set of natural numbers $\{1,2,3, \ldots\}$, and let $\mathbb{R}$ denote the set of real numbers. Let $Y$ denote the closed interval $[0,1]$, and let the set $Y^{\mathbb{N}}$ be denoted by $X$. Then, $X$ is the domain of utility sequences that we are interested in. Hence, $x \equiv\left(x_{1}, x_{2}, \ldots\right) \in X$ if and only if $x_{n} \in[0,1]$ for all $n \in \mathbb{N}$.

For $y, z \in \mathbb{R}^{\mathbb{N}}$, we write $y \geq z$ if $y_{i} \geq z_{i}$ for all $i \in \mathbb{N} ;$ and, we write $y>z$ if $y \geq z$, and $y \neq z$.

A social welfare relation (SWR) is a binary relation, $\succsim$, on $X$, which is reflexive and transitive (a pre-ordering). ${ }^{11}$ We associate with $\succsim$ its symmetric

\footnotetext{
${ }^{10}$ The study by Brock (1970) uses a "consistency axiom" which, together with the independence axiom, actually implies a continuity restriction on the underlying preferences. A more recent study by Asheim and Tungodden (2001) also uses a continuity axiom. This point is discussed in detail in Section 4.

${ }^{11}$ In the economics literature, a pre-ordering is often refered to as a "partial ordering" or as a "quasi ordering". However, in the mathematics literature, the term "partial ordering" refers to a binary relation which is transitive and antisymmetric. To avoid confusion, we use the mathematical terminology, since the term "pre-order" is never used in any other sense in either discipline. Incidentally, our usage coincides with the terminology introduced
} 
and asymmetric components in the usual way. Thus, we write $x \sim y$ when $x \succsim y$ and $y \succsim x$ both hold; and, we write $x \succ y$ when $x \succsim y$ holds, but $y \succsim x$ does not hold. A social welfare ordering (SWO) is a binary relation, $\succsim$, on $X$, which is complete ${ }^{12}$ and transitive (a complete pre-ordering).

A SWR $\succsim_{A}$ is a subrelation to a SWR $\succsim_{B}$ if (a) $x, y \in X$ and $x \succsim_{A} y$ implies $x \succsim_{B} y$; and (b) $x, y \in X$ and $x \succ_{A} y$ implies $x \succ_{B} y$. A SWO $\succsim_{A}$ is compatible with a SWR $\succsim_{B}$ if and only if $\succsim_{B}$ is a subrelation to $\succsim_{A}$.

Given $x \in X$, and $N \in \mathbb{N}$, let us denote by $x(N)$ the vector consisting of the first $N$ elements of $x$ and by $x[N]$ the sequence from term $(N+1)$ onwards. So, $x(N)=\left(x_{1}, x_{2}, \ldots, x_{N}\right)$ and $x[N]=\left(x_{N+1}, x_{N+2}, \ldots\right)$. The sequence $\left(x_{1}, x_{2}, \ldots, x_{N}, 0,0, \ldots\right)$ is denoted by $(x(N), 0[N])$. Given a vector $x(N)$, we use $I(x(N))$ to denote $\left(x_{1}+\cdots+x_{N}\right)$.

\section{The Utilitarian Social Welfare Relation}

In this section, we introduce a new definition of a utilitarian social welfare relation, and provide an axiomatic characterization of it in terms of the Anonymity, Pareto and Partial Unit Comparability axioms. We also relate our utilitarian SWR to the Suppes-Sen grading principle, which is characterized in terms of the first two of these axioms.

Let us define a binary relation $\succsim_{U}$ on $X$ by:

$$
\begin{gathered}
x \succsim_{U} y \text { if and only if there is } N \in \mathbb{N} \text {, such that } \\
(I(x(N)), x[N]) \geq(I(y(N)), y[N])
\end{gathered}
$$

It is easy to check that $\succsim_{U}$ is reflexive and transitive on $X$, so it is a SWR. We will call this SWR utilitarian. Note that the utilitarian social welfare relation ranks only those infinite utility streams which are "Pareto comparable" beyond a finite horizon, and applies standard utilitarian principles up to that finite horizon.

The SWR ${ }_{U}$ satisfies the following two desirable properties:

$$
\begin{gathered}
\text { (a)If } x, y \in X \text { and } N \in \mathbb{N} \text { and }(I(x(N)), x[N]) \geq(I(y(N)), y[N]) \\
\text { then } x \succsim_{U} y
\end{gathered}
$$

in Debreu (1959).

${ }^{12}$ Since completeness implies reflexivity, a social welfare ordering is a social welfare relation, which is complete. 
and

$$
\begin{gathered}
\text { (b)If } x, y \in X \text { and } N \in \mathbb{N} \text { and }(I(x(N)), x[N])>(I(y(N)), y[N]) \\
\text { then } x \succ_{U} y
\end{gathered}
$$

\subsection{Axiomatic Characterization of the Utilitarian SWR}

Our objective is to establish an axiomatic characterization of the utilitarian SWR. To this end, consider the following two axioms on a SWR $\succsim$, which are fairly straightforward, and therefore require no explanation.

Axiom 1 (Pareto) If $x, y \in X$, and there is some $j \in \mathbb{N}$, such that $x_{j}>y_{j}$, while $x_{k} \geq y_{k}$ for all $k \neq j$, then $x \succ y$.

Axiom 2 (Anonymity) If $x, y$ are in $X$, and there exist $i, j$ in $\mathbb{N}$, such that $x_{i}=y_{j}$ and $x_{j}=y_{i}$, while $x_{k}=y_{k}$ for all $k \in \mathbb{N}$, such that $k \neq i, j$, then $x \sim y$.

The next axiom is an adaptation to the infinite domain of the standard assumption of unit interpersonal comparability used in social choice theory (see, for instance, Sen(1977), d'Aspremont and Gevers (1977), Roberts (1980), Basu (1983)), expressed as an invariance axiom. ${ }^{13}$

Axiom 3 (Partial Unit Comparability) If $x, y \in X, \alpha \in \mathbb{R}^{\mathbb{N}}$ and $N \in \mathbb{N}$ satisfy:

$$
(x(N), x[N]) \succsim(y(N), x[N])
$$

and:

$$
(x(N), x[N])+\alpha \in X,(y(N), x[N])+\alpha \in X
$$

then they must also satisfy:

$$
(x(N), x[N])+\alpha \succsim(y(N), x[N])+\alpha
$$

\footnotetext{
${ }^{13}$ Maskin (1978) uses the weaker "full comparability axiom" in which one demands invariance only for a common change of origin and a common change of scale for all agents. He is able to characterize utilitarianism (in finite societies) by using this "full comparability axiom" [instead of the stronger "unit comparability axiom" in d'Aspremont-Gevers(1977)] by exploiting in addition a continuity axiom.
} 
Remark 1 (i) The Unit Comparability axiom (on the infinite domain) asserts that preferences are invariant to changes in the origins of the utility indices used in the various periods; it is also invariant to a common change in the scale (by a positive factor) of the utility indices used in the various periods. It would be formally stated as follows. ${ }^{14}$

(Unit Comparability)Let $a, b, a^{\prime}, b^{\prime} \in X$ be such that there exists a sequence of real numbers $\left\{\alpha_{n}\right\}$ and a positive real number $\beta$ satisfying for all $n \in \mathbb{N}$,

$$
a_{n}^{\prime}=\alpha_{n}+\beta a_{n} ; b_{n}^{\prime}=\alpha_{n}+\beta b_{n}
$$

Then,

$$
a \succsim b \text { if and only if } a^{\prime} \succsim b^{\prime}
$$

(ii) Axiom 3 is weaker than the Unit Comparability axiom, since we insist on the invariance with respect to changes in origin only in comparing utility streams in which the streams are identical from a certain point onwards. ${ }^{15}$

It is fairly straightforward to check that if the utilitarian SWR $\succsim_{U}$ is a subrelation to a SWR $\succsim$, then $\succsim$ must satisfy the Pareto, Anonymity and Partial Unit Comparability Axioms. What is not so obvious is that if $\succsim$ is any SWR satisfying these three axioms, then the utilitarian SWR $\succsim_{U}$ must be a subrelation to $\succsim$. Essential to this complete characterization theorem is a technical lemma, which should be of independent interest. This intermediate result provides a characterization of the indifference classes (of SWRs satisfying the three axioms) on the subset of $X$ consisting of utility streams with at most a finite number of non-zero entries.

Define $X^{0}=\{x \in X: x$ has at most a finite number of non-zero elements $\}$. Note that for $x \in X^{0}$, the sum $\sum_{n=1}^{\infty} x_{n}$ is well-defined; we denote it by $\sigma(x)$. For $x \in X^{0}$, the decreasing rearrangement of $x$ is clearly also well-defined; we denote it by $\hat{x}$. Define $m(\hat{x})=\min \left\{N \in \mathbb{N}: x_{n}=0\right.$ for all $n \geq N\}$.

Lemma 1 (i)Suppose a $S W R \succsim$ satifies Axioms 2,3. If $x, y \in X^{0}$, and $\sigma(x)=\sigma(y)$, then $x \sim y$. (ii) Suppose a SWR $\succsim$ satisfies Axioms 1-3. If $x, y \in X^{0}$ and $x \sim y$, then $\sigma(x)=\sigma(y)$.

\footnotetext{
${ }^{14}$ This axiom has been used by Lauwers (1997) in his axiomatic characterization of discounted utilitarianism.

${ }^{15}$ Weaker than the above partial unit comparability axiom is the independence postulate, introduced by Debreu (1960) in the finite-horizon context, and studied by Koopmans (1960) and Koopmans, Diamond and Williamson (1964) in an infinite-horizon context, in their studies on the representation of preferences by additively separable utility functions.
} 
Proof. (i)Let $m=\max \{m(\hat{x}), m(\hat{y})\}$. Then, for $n \geq m$, we have $\hat{x}_{n}=$ $\hat{y}_{n}=0$. We prove the result by induction on $m$. For $m=1$, we have $x=y=0$, and the result is trivially true. Suppose, next, that the result is true for $m=1, \ldots, M$, where $M \in \mathbb{N}$. We want to prove that the result is true for $m=M+1$.

If $\hat{x}=\hat{y}$, then $x \sim y$ by the Anonymity Axiom. So, we need only consider the case in which $\hat{x} \neq \hat{y}$. Then, there exist some $i, j \in\{1, \ldots, M\}$, such that $\hat{x}_{i}>\hat{y}_{i}$ and $\hat{x}_{j}<\hat{y}_{j}$. Define $\alpha_{n}=\min \left\{\hat{x}_{n}, \hat{y}_{n}\right\}, x_{n}^{\prime}=\hat{x}_{n}-\alpha_{n}$ and $y_{n}^{\prime}=\hat{y}_{n}-\alpha_{n}$ for all $n \in \mathbb{N}$. Notice that $x^{\prime}, y^{\prime} \in X^{0}$, and $x^{\prime} \leq \hat{x}$, while $y^{\prime} \leq \hat{y}$. Further, we have $\sigma\left(x^{\prime}\right)=\sigma\left(y^{\prime}\right)$. Also, $y_{i}^{\prime}=0$, and $x_{j}^{\prime}=0$; furthermore, $x_{n}^{\prime}=y_{n}^{\prime}=0$ for all $n \geq M+1$. It follows that $m\left(\hat{x}^{\prime}\right) \leq M$ and $m\left(\hat{y}^{\prime}\right) \leq M$; consequently $\max \left\{m\left(\hat{x}^{\prime}\right), m\left(\hat{y}^{\prime}\right)\right\} \leq M$. Thus by the induction hypothesis, $\hat{x}^{\prime} \sim \hat{y}^{\prime}$, and by the Anonymity axiom, we must have $x^{\prime} \sim y^{\prime}$. Now, using the Partial Unit Comparability axiom, we get $\hat{x} \sim \hat{y}$. The Anonymity Axiom can now be employed again to conclude that $x \sim y$. This completes the proof of (i) by induction.

(ii) Suppose, on the contrary, there exist $x, y \in X^{0}$ satisfying $x \sim y$, but $\sigma(x) \neq \sigma(y)$. Without loss of generality, we may suppose that $\sigma(x)>\sigma(y)$; denote $[\sigma(x)-\sigma(y)]$ by $d$. Clearly, there exists $N \in \mathbb{N}$, such that $x_{i}=y_{i}=0$ for all $i>N$. Then, we have $0<d \leq N$. Define $x^{\prime} \in \mathbb{R}^{\mathbb{N}}$ as follows:

$$
\left.\begin{array}{lll}
x_{i}^{\prime}=y_{i} & \text { for } & i=1, \ldots, N \\
x_{i}^{\prime}=(d / N) & \text { for } & i=N+1, \ldots, 2 N \\
x_{i}^{\prime}=0 & \text { for } & i>2 N
\end{array}\right\}
$$

Clearly, $x^{\prime} \in X$ and $x^{\prime}>y$, so by the Pareto axiom, $x^{\prime} \succ y$. It is also clear that $x^{\prime} \in X^{0}$, and $\sigma\left(x^{\prime}\right)=\sigma(y)+d=\sigma(x)$. Thus, by part (i) of the Lemma, we have $x^{\prime} \sim x$. Since $x \sim y$, we must have $x^{\prime} \sim y$, a contradiction, which establishes (ii).

Remark 2 The proof of Lemma 1(i) follows the method used by Milnor (1954) in his axiomatic characterization of the Laplace criterion in games against nature. This idea has also been used in the context of social choice theory in characterizing utilitarianism in a society with a finite number of agents by d'Aspremont and Gevers (1977). For finite agent societies, a diagrammatic exposition of their result is provided in Blackorby, Donaldson and Weymark (1984).

We now present our characterization result regarding the utilitarian SWR $\succsim_{U}$. 
Theorem 1 The utilitarian $S W R \succsim_{U}$ is a subrelation to a $S W R \succsim$ if and only if $\succsim$ satisfies Axioms 1,2 and 3.

Proof. (Necessity) Suppose the utilitarian SWR ${ }_{U}$ is a subrelation to a SWR $\succsim$. We need to verify that $\succsim$ satisfies Axioms $1-3$. To verify that $\succsim$ satisfies the Pareto axiom, let $x, y \in X$, such that there is some $j \in \mathbb{N}$ for which $x_{j}>y_{j}$, while $x_{k} \geq y_{k}$ for all $k \neq j$. Then, clearly, we have $(I(x(j)), x[j])>(I(y(j)), y[j])$, so $x \succ_{U} y$ by $(3)$. Since $\succsim_{U}$ is a subrelation to $\succsim$, we have $x \succ y$. To verify that $\succsim$ satisfies the Anonymity axiom, let $x, y \in X$, and $i, j \in \mathbb{N}$ be such that $x_{i}=y_{j}$ and $x_{j}=y_{i}$, while $x_{k}=y_{k}$ for all $k \in \mathbb{N}$, such that $k \neq i, j$. Then, defining $N=\max \{i, j\}$, we have $(I(x(N)), x[N])=(I(y(N)), y[N])$, so that $x \succsim_{U} y$ and $y \succsim_{U} x$ by (2). Since $\succsim_{U}$ is a subrelation to $\succsim$, we have $x \succsim y$ and $y \succsim x$. Thus, $x \sim y$, as required. To verify the Partial Unit Comparability axiom, let $x, y \in X, \alpha \in \mathbb{R}^{\mathbb{N}}$ and $N \in \mathbb{N}$ satisfy:

$$
(x(N), x[N]) \succsim(y(N), x[N])
$$

and:

$$
(x(N), x[N])+\alpha \in X,(y(N), x[N])+\alpha \in X
$$

We claim that $I(x(N)) \geq I(y(N))$. For if $I(x(N))<I(y(N))$, then by (3), we have $(y(N), x[N]) \succ_{U}(x(N), x[N])$. Since $\succsim_{U}$ is a subrelation to $\succsim$, we must then have $(y(N), x[N]) \succ(x(N), x[N])$, a contradiction to (9), which establishes our claim. Thus, we have:

$I(x(N)+\alpha(N))=I(x(N))+I(\alpha(N)) \geq I(y(N))+I(\alpha(N))=I(y(N)+\alpha(N))$

and:

$$
(I(x(N)+\alpha(N)), x[N]+\alpha[N]) \geq(I(y(N)+\alpha(N)), x[N]+\alpha[N])
$$

so that $(x(N)+\alpha(N), x[N]+\alpha[N]) \succsim_{U}(y(N)+\alpha(N), x[N]+\alpha[N])$ by $(2)$ and (10). Since $\succsim_{U}$ is a subrelation to $\succsim$, we have $(x(N)+\alpha(N), x[N]+$ $\alpha[N]) \succsim(y(N)+\alpha(N), x[N]+\alpha[N])$. Thus (6) is satisfied, and the Partial Unit Comparability axiom is verified.

(Sufficiency) Suppose a SWR $\succsim$ satisfies Axioms 1,2 and 3. We want to show that $\succsim_{U}$ is a subrelation to $\succsim$. To this end, let $x, y \in X$, and suppose $x \succ_{U} y$. Then, by (1), there is some $N^{\prime} \in \mathbb{N}$, such that $\left(I\left(x\left(N^{\prime}\right)\right), x\left[N^{\prime}\right]\right)>$ $\left(I\left(y\left(N^{\prime}\right)\right), y\left[N^{\prime}\right]\right)$. So, there is $N \geq N^{\prime}$ such that:

$$
I(x(N)>I(y(N) \text { and } x[N]) \geq y[N])
$$


We want to prove that $x \succ y$. Denote $[I(x(N))-I(y(N))]$ by $d$; then $d>0$. Define:

$$
d_{i}=\left(1-y_{i}\right) d /[N-I(y(N))] \text { for } i=1, \ldots, N
$$

Note that $0 \leq d_{i} \leq\left(1-y_{i}\right)$ for $i=1, \ldots, N$, and:

$$
\sum_{i=1}^{N} d_{i}=d
$$

Now, define $x^{\prime}, y^{\prime}, x^{\prime \prime}, y^{\prime \prime}$ as follows: $x^{\prime}=(x(N), y[N]), y^{\prime}=\left(y_{1}+d_{1}, \ldots, y_{N}+\right.$ $\left.d_{N}, y[N]\right), x^{\prime \prime}=(x(N), 0[N]), y^{\prime \prime}=\left(y_{1}+d_{1}, \ldots, y_{N}+d_{N}, 0[N]\right)$. Clearly, $x^{\prime}, y^{\prime} \in X$ and $x^{\prime \prime}, y^{\prime \prime} \in X^{0}$.

Note that $\sigma\left(x^{\prime \prime}\right)=\sigma\left(y^{\prime \prime}\right)$, since :

$$
\sum_{i=1}^{N}\left(y_{i}+d_{i}\right)=\sum_{i=1}^{N} y_{i}+d=\sum_{i=1}^{N} y_{i}+[I(x(N))-I(y(N))]=I(x(N))
$$

Using (13) and Lemma 1, we must have $x^{\prime \prime} \sim y^{\prime \prime}$. Using the partial unit comparability axiom, it follows that $x^{\prime} \sim y^{\prime}$. By the Pareto Axiom, we obtain $y^{\prime} \succ y$, and $x \succsim x^{\prime}$. Thus, we must have $x \succ y$ by transitivity of $\succsim$.

Now, let $x, y \in X$, and suppose $x \succsim_{U} y$. Then, by (1), there is some $N \in \mathbb{N}$, such that:

$$
(I(x(N)), x[N]) \geq(I(y(N)), y[N])
$$

We want to prove that $x \succsim y$. If in fact we have $(I(x(N)), x[N])>$ $(I(y(N)), y[N])$, then $x \succ_{U} y$, so that $x \succ y$ must hold, as proved above, and we are done. So, we are left with the case in which $(I(x(N)), x[N])=$ $(I(y(N)), y[N])$. In this case, define $\bar{x}, \bar{y}$ as follows: $\bar{x}=(x(N), 0[N]), \bar{y}=$ $(y(N), 0[N])$. Clearly, $\bar{x}, \bar{y} \in X^{0}$. Since $I(x(N))=I(y(N))$, we have $\sigma(\bar{x})=$ $\sigma(\bar{y})$ and by Lemma $1, \bar{x} \sim \bar{y}$. Since $x[N]=y[N]$, the partial unit comparability axiom implies that $x \sim y$.

It is useful to view the above characterization result as saying that the utilitarian SWR is the least restrictive SWR among all SWRs satisfying the Pareto, Anonymity and Partial Unit Comparability axioms. For this purpose, it is convenient to identify a binary relation on $X$ with its graph in $X^{2} .{ }^{16}$

\footnotetext{
${ }^{16}$ Recall that a binary relation is often defined precisely by specifying its graph.
} 
Denote by $P\left(X^{2}\right)$ the set of all subsets of $X^{2}$. Note that the binary relation $\subset$ ("subset of") is a pre-order on $P\left(X^{2}\right)$. If $\succsim$ is any binary relation on $X$, its graph:

$$
S(\succsim)=\left\{(x, y) \in X^{2}: x \succsim y\right\}
$$

is a subset of $X^{2}$, and consequently, $S(\succsim)$ is an element of $P\left(X^{2}\right)$.

We look at the subset $Q\left(X^{2}\right)$ of $P\left(X^{2}\right)$ consisting of the graphs of those pre-orders on $X$ which satisfy Axioms 1-3. Formally, $Q\left(X^{2}\right)$ is the subset of $P\left(X^{2}\right)$, defined by:

$$
\begin{gathered}
Q\left(X^{2}\right)=\left\{S(\succsim) \in P\left(X^{2}\right): \succsim \text { is a pre }- \text { order on } X\right. \\
\text { satisfying Axioms } 1-3\}
\end{gathered}
$$

Using Theorem 1, we have $S\left(\succsim_{U}\right) \in Q\left(X^{2}\right)$, and if $\succsim$ is any pre-order on $X$ satisfying Axioms 1-3, then $S\left(\succsim_{U}\right) \subset S\left(\succsim_{)}\right.$. Thus, $S\left(\succsim_{U}\right)$ is a least element of $Q\left(X^{2}\right)$ in terms of the pre-order $\subset .{ }^{17}$ In this sense, the utilitarian SWR is the least restrictive pre-order satisfying Axioms 1-3.

It follows from this result that the graph of $\succsim_{U}$ is in fact the intersection of the graphs of all pre-orders on $X$ satisfying Axioms 1-3. Let us define:

$$
S=\bigcap_{S(\succsim) \in Q\left(X^{2}\right)} S(\succsim)
$$

Then, by definition, we have $S \subset S(\succsim)$ for every $S(\succsim) \in Q\left(X^{2}\right)$. Since $S\left(\succsim_{U}\right) \in Q\left(X^{2}\right)$, we have $S \subset S\left(\succsim_{U}\right)$. On the other hand, since $S\left(\succsim_{U}\right)$ is a least element of $Q\left(X^{2}\right)$ in terms of the pre-order $\subset$, we have $S\left(\succsim_{U}\right) \subset S(\succsim)$ for all $S(\succsim) \in Q\left(X^{2}\right)$. That is, $S\left(\succsim_{U}\right) \subset S$. Thus, we have:

$$
S\left(\succsim_{U}\right)=S=\bigcap_{S(\succsim) \in Q\left(X^{2}\right)} S(\succsim)
$$

\subsection{Comparison with the Grading Principle}

Recall that the Suppes-Sen grading principle is the binary relation $\succsim_{S}$ defined on $X$ as follows:

$$
\begin{gathered}
x \succsim_{S} y \text { if and only if there is a finite permutation } \\
\pi \text { of } \mathbb{N} \text {, such that } x(\pi) \geq y
\end{gathered}
$$

\footnotetext{
${ }^{17}$ We use here the standard mathematical definition of a "least element" of a set, given a pre-order on that set. See, for example, Debreu(1959, p.8).
} 
Asheim, Buchholz and Tungodden (2001, p.256) have characterized it in terms of the Anonymity and Pareto axioms as follows.

Proposition 1 A binary relation $\succsim$ on $X$ satisfies Axioms 1 and 2 if and only if the grading principle $\succsim_{S}$ is a subrelation to $\succsim$.

The grading principle does not satisfy the partial unit comparability axiom, as can be seen from the following example. Let $x=(0.5,0.4,0.1,0.1, \ldots)$ and $y=(0.3,0.8,0.1,0.1, \ldots)$. Then, $x$ and $y$ are non-comparable by the Suppes-Sen grading principle, since there is no finite permuation of $x$ which is $\geq y$, and there is no finite permutation of $y$ which is $\geq x$. However, if we increase the utility origin of the first period by 0.1 , and reduce the utility origin in the second period by 0.1 , we obtain the vectors $\bar{x}=(0.6,0.3,0.1,0.1, \ldots)$ and $\bar{y}=(0.4,0.7,0.1,0.1, \ldots)$. Now, permuting the first two periods of the vector $\bar{y}$, and denoting the resulting vector by $\bar{y}(\pi)$, we see that $\bar{y}(\pi)>\bar{x}$, so that $\bar{y}$ is preferred to $\bar{x}$ according to the Suppes-Sen grading principle. Thus, the Suppes-Sen grading principle violates the partial unit comparability axiom.

The characterizations of the grading principle and the utilitarian SWR allow us to obtain a social welfare ordering (a complete pre-order) compatible with the utilitarian SWR, which satisfies Anonymity and the Pareto axioms. Since the binary relation ${ }_{U}$ satisfies the Anonymity and Pareto axioms (by Theorem 1), the grading Principle $\succsim_{S}$ is clearly a subrelation to $\succsim_{U}$ (by Proposition 1). Thus, by Theorem 2 of Svensson (1980, p.1253), there is a complete pre-order $\succsim$ compatible with ${ }_{U}$, which satisfies the Pareto and Anonymity Axioms.

\section{The overtaking criterion SWRs}

The standard method of comparing utility streams in infinite-horizon intertemporal allocation models, while respecting the equal treatment of all generations, is by employing the overtaking criterion. The resulting pre-order is a generalization of the one used by Ramsey (1928), and was proposed independently by Atsumi (1965) and von Weizsacker (1965) in their studies on optimal economic growth.

It would be useful to discuss the merits of our (less restrictive) utilitarian 
SWR with the (more restrictive) overtaking SWRs. ${ }^{18}$ For this purpose, it would be convenient to have axiomatic characterizations of the overtaking SWRs which are directly comparable to our characterization of the utilitarian SWR. Unfortunately, the characterizations of the overtaking SWRs provided by Brock (1970) and more recently by Asheim and Tungodden (2002) use axiom sets which make such a direct comparison difficult. ${ }^{19}$ In this section, we provide an axiomatic characterization of the overtaking SWRs, which will facilitate such a comparison.

We will show that the overtaking SWRs can be characterized in terms of Axioms 1-3 and an additional "consistency" axiom. This consistency axiom is similar in spirit to Axiom 3 used in Brock (1970), who says that it "captures the notion that decisions on infinite programs are consistent with decisions on finite programs of length $n$ if $n$ is large enough."

The partial unit comparability axiom together with the consistency axiom imply a continuity requirement on the SWR, similar to that used by Asheim and Tungodden (2002) in their axiomatic characterization of the overtaking SWRs. ${ }^{20}$ Thus, in terms of the axiomatics, the difference between the overtaking SWRs and our utilitarian SWR can be traced to imposing or not imposing a continuity requirement on preferences. In this regard, the current work can be seen as a continuation of the study in Basu and Mitra (2002), where we deliberately refrained from imposing any continuity axiom. Axioms on the continuity of preferences in infinite-dimensional spaces have been the most controversial in the literature in this area, since the topology in which such continuity is assumed determines to a large extent the nature of allowable preferences. These restrictions arise largely from mathematical necessity and do not necessarily reflect any underlying ethical or economic principle.

\subsection{Axiomatic Characterization of the Overtaking SWRs}

There are two definitions of the overtaking criterion commonly in use. We will define each in turn and provide their axiomatic characterizations. Let

\footnotetext{
${ }^{18} \mathrm{~A}$ more appropriate way to describe them would be "the SWRs induced by the overtaking criterion and the catching-up criterion". The precise definitions are given in Section 4.1 .

${ }^{19}$ In particular, neither of these papers uses the partial unit comparability axiom directly.

${ }^{20}$ This is discussed in detail in the discussion following Theorems 2 and 3.
} 
us define ${ }^{21}$ a binary relation $\succsim_{C}$ on $X$ by:

$$
\begin{gathered}
x \succsim_{C} y \text { if and only if there is } \bar{N} \in \mathbb{N} \text {, such that } \\
\qquad I(x(N)) \geq I(y(N)) \text { for all } N \geq \bar{N}
\end{gathered}
$$

It is easy to check that $\succsim_{C}$ is reflexive and transitive on $X$, so it is a SWR. We will call it the catching up SWR.

The SWR $\succsim_{C}$ satisfies the following two properties:

$$
\begin{gathered}
(a) \text { If } x, y \in X \text { and } \bar{N} \in \mathbb{N} \text { and } I(x(N)) \geq I(y(N)) \\
\text { for all } N \geq \bar{N}, \text { then } x \succsim_{C} y
\end{gathered}
$$

and

$$
\begin{gathered}
\text { (b)If } x, y \in X \text { and } \bar{N} \in \mathbb{N} \text { and } I(x(N)) \geq I(y(N)) \\
\text { for all } N \geq \bar{N} \text {, and } I(x(N))>I(y(N)) \\
\text { for a subsequence of } N \geq \bar{N}, \text { then } x \succ_{C} y
\end{gathered}
$$

We can obtain an axiomatic characterization of the catching up SWR in terms of Axioms 1-3 of the previous section, and an additional strong consistency axiom, which we now state.

Axiom 4 (Strong Consistency) For $x, y \in X$,

(a)If there is $\bar{N} \in \mathbb{N}$, such that $(x(N), 0[N]) \succsim(y(N), 0[N])$

$$
\text { for all } N \geq \bar{N} \text {, then } x \succsim y
$$

(b)If there is $\bar{N} \in \mathbb{N}$, such that $(x(N), 0[N]) \succsim(y(N), 0[N])$

$$
\begin{gathered}
\text { for all } N \geq \bar{N} \text {, with }(x(N), 0[N]) \succ(y(N), 0[N]) \\
\text { for a subsequence of } N \geq \bar{N}, \text { then } x \succ y
\end{gathered}
$$

The characterization result can be stated and proved as follows.

Theorem $2 A S W R \succsim$ satisfies Axioms 1-4 if and only if $\succsim_{C}$ is a subrelation to $\succsim$.

\footnotetext{
${ }^{21}$ This definition is used by Svensson (1980).
} 
Proof. (i) Suppose a SWR $\succsim$ satisfies Axioms 1-4. Let $x, y \in X$ and $x \succsim_{C} y$. Then, by (14), there is $\bar{N} \in \mathbb{N}$ such that:

$$
I(x(N)) \geq I(y(N)) \text { for all } N \geq \bar{N}
$$

Using (18) and (2), we get $(x(N), 0[N]) \succsim_{U}(y(N), 0[N])$ for all $N \geq$ $\bar{N}$. Thus, for each $N \geq \bar{N}$, we have $(x(N), 0[N]) \succsim(y(N), 0[N])$ by using Theorem 1. Using Axiom 4(a), it follows that $x \succsim y$.

Next, let $x, y \in X$ and $x \succ_{C} y$. Then, $x \succsim_{C} y$ holds, but $y \succsim_{C} x$ does not hold. Using $x \succsim_{C} y$, there is $\bar{N} \in \mathbb{N}$ such that (18) holds; further (18) must hold with strict inequality for a subsequence $N^{s}$ of $N$, otherwise $y \succsim_{C} x$ would also hold. Thus, using (2) and (3), we must have $(x(N), 0[N]) \succsim_{U}$ $(y(N), 0[N])$ for all $N \geq \bar{N}$, with $\left(x\left(N^{s}\right), 0\left[N^{s}\right]\right) \succ_{U}\left(y\left(N^{s}\right), 0\left[N^{s}\right]\right)$ for the subsequence $N^{s}$. Consequently, we have $(x(N), 0[N]) \succsim(y(N), 0[N])$ for each $N \geq \bar{N}$, with $(x(N), 0[N]) \succ(y(N), 0[N])$ for the subsequence $N^{s}$.Using Axiom 4(b), it follows that $x \succ y$.

(ii) Suppose that the social welfare relation $\succsim_{C}$ is a subrelation to a SWR $\succsim$. It can be verified, by following the method used in the proof of Theorem 1, that the SWR $\succsim$ satisfies Axioms 1-3. We now check Axiom 4 as follows.

(a) Suppose $x, y \in X$ and there is $\bar{N} \in \mathbb{N}$,such that $(x(N), 0[N]) \succsim$ $(y(N), 0[N])$ for all $N \geq \bar{N}$. Pick any $N \geq \bar{N}$. We claim that:

$$
I(x(N)) \geq I(y(N))
$$

For if (19) is violated, then $(y(N), 0[N]) \succ_{U}(x(N), 0[N])$ by (3). Using Theorem 1, we obtain $(y(N), 0[N]) \succ(x(N), 0[N])$, a contradiction. This establishes our claim (19). Using (19) and (15), we get $x \succsim_{C} y$, and since $\succsim_{C}$ is a subrelation to $\succsim$, we get $x \succsim y$. This verifies Axiom 4(a).

(b) Suppose $x, y \in X$ and there is $\bar{N} \in \mathbb{N}$, such that $(x(N), 0[N]) \succsim$ $(y(N), 0[N])$ for all $N \geq \bar{N}$, with $\left(x\left(N^{s}\right), 0\left[N^{s}\right]\right) \succ\left(y\left(N^{s}\right), 0\left[N^{s}\right]\right)$ for a subsequence $N^{s}$ of $N \geq \bar{N}$. Pick any $N \geq \bar{N}$. We can use the method used above to verify Axiom 4(a), to obtain (19), with strict inequality in (19) holding for the subsequence $N^{s}$ of $N$. By (16), $x \succ_{C} y$ holds and since $\succsim_{C}$ is a subrelation to $\succsim$, we obtain $x \succ y$, verifying Axiom 4(b).

Asheim and Tungodden (2002) use a "Strong Preference Continuity" axiom in their characterization of the catching up SWR. This axiom is stated as follows.

(Strong Preference Continuity) Suppose $x, y \in X$ and $\bar{N} \in \mathbb{N}$, satisfy:

$$
(y(N), x[N]) \succsim(x(N), x[N]) \text { for all } N \geq \bar{N}
$$


and:

$$
(y(N), x[N]) \succ(x(N), x[N]) \text { for a subsequence of } N \geq \bar{N}
$$

then $y \succ x$.

This axiom is implied by Axiom 3 and Axiom 4. Using Axiom 3 and (Ca), $(\mathrm{Cb})$, we have:

$$
(y(N), 0[N]) \succsim(x(N), 0[N]) \text { for all } N \geq \bar{N}
$$

and:

$$
(y(N), 0[N]) \succ(x(N), 0[N]) \text { for a subsequence of } N \geq \bar{N}
$$

Thus, by Axiom 4, we obtain $y \succ x$.

To see that this is indeed a strong continuity requirement, define the sequence of infinite utility streams $z^{s}=(y(s), x[s])$ for each $s \in \mathbb{N}$. Then, we see that $y$ is the (pointwise) limit of $z^{s}$ as $s \rightarrow \infty$. Thus, the axiom says that if $z^{s} \succsim x$ for all $s$ sufficiently large, with $z^{s} \succ x$ for a subsequence of $s$, then the pointwise limit of $z^{s}$ (namely, $\left.y\right) \succ x$. Quite apart from the fact that preferences cannot be reversed in the limit, this in fact demands that strict preference prevail in the limit. ${ }^{22}$

The alternative version of the overtaking criterion ${ }^{23}$ can be formalized as follows. Let us define the binary relation $\succsim_{O}$ by:

$$
\begin{gathered}
x \succsim_{o} y \text { if and only if } \\
\text { either }(i) \exists \bar{N} \in \mathbb{N} \text {, such that } I(x(N))>I(y(N)) \text { for all } N \geq \bar{N} \\
\text { or }(\text { ii }) \exists \bar{N} \in \mathbb{N} \text {, such that } I(x(N))=I(y(N)) \text { for all } N \geq \bar{N}
\end{gathered}
$$

It is easy to check that $\succsim_{O}$ is reflexive and transitive on $X$, so it is a SWR. We will call it the overtaking SWR.

It can be verified that the SWR $\succsim o$ satisfies the following two properties:

$$
\begin{gathered}
\text { (a) } x \succ_{O} y \text { if and only if there is } \bar{N} \in \mathbb{N}, \\
\text { such that } I(x(N))>I(y(N)) \text { for all } N \geq \bar{N}
\end{gathered}
$$

\footnotetext{
${ }^{22}$ Asheim and Tungodden (2002) use the word "strong" here because strict preference in the limit is based on strict preference holding only along a subsequence of $s$. But, clearly, this continuity requirement is very strong for other reasons as well.

${ }^{23}$ This is the version used by Atsumi (1965), von Weizsacker (1965) and Brock (1970).
} 
and

$$
\begin{gathered}
\text { (b) } x \sim_{O} y \text { if and only if there is } \bar{N} \in \mathbb{N}, \\
\text { such that } I(x(N))=I(y(N)) \text { for all } N \geq \bar{N}
\end{gathered}
$$

It follows that :

$$
\text { (i) } x \succ_{O} y \Longrightarrow x \succ_{C} y ;(i i) x \sim_{O} y \Longrightarrow x \sim_{C} y
$$

We can obtain an axiomatic characterization of the strong overtaking SWR in terms of Axioms 1-3 of the previous section, and an additional weak consistency axiom, which we now state.

Axiom 5 (Weak Consistency) For $x, y \in X$,

(a) If there is $\bar{N} \in \mathbb{N}$, such that $(x(N), 0[N]) \sim(y(N), 0[N])$

$$
\text { for all } N \geq \bar{N} \text {, then } x \sim y
$$

(b) If there is $\bar{N} \in \mathbb{N}$, such that $(x(N), 0[N]) \succ(y(N), 0[N])$

$$
\text { for all } N \geq \bar{N} \text {, then } x \succ y
$$

The characterization result for the overtaking SWR can be stated as follows. The proof, which is similar to the proof of Theorem 2, is omitted.

Theorem $3 A S W R \succsim$ satisfies Axioms 1-3 and 5 if and only if o $_{0}$ is a subrelation to $\succsim$.

Asheim and Tungodden (2002) use a "Weak Preference Continuity" axiom in their characterization of the overtaking SWR. This axiom is stated as follows.

(Weak Preference Continuity) Suppose $x, y \in X$ and $\bar{N} \in \mathbb{N}$, satisfy:

$$
(y(N), x[N]) \succ(x(N), x[N]) \text { for all } N \geq \bar{N}
$$

then $y \succ x$.

This axiom is implied by Axiom 3 and Axiom 5. Using Axiom 3 and $(\mathrm{Cw})$, we have:

$$
(y(N), 0[N]) \succsim(x(N), 0[N]) \text { for all } N \geq \bar{N}
$$


Further:

$$
(x(N), 0[N]) \succsim(y(N), 0[N])
$$

cannot hold for any $N \geq \bar{N}$. For if it did hold for some $N \geq \bar{N}$, then by Axiom 3, we would get:

$$
(x(N), x[N]) \succsim(y(N), x[N])
$$

for that $N$. But, this would contradict $(\mathrm{Cw})$. Thus, we have:

$$
(y(N), 0[N]) \succ(x(N), 0[N]) \text { for all } N \geq \bar{N}
$$

and, by Axiom 5, we obtain $y \succ x$.

Define the sequence of infinite utility streams $z^{s}=(y(s), x[s])$ for each $s \in \mathbb{N}$. Then, we see that $y$ is the (pointwise) limit of $z^{s}$ as $s \rightarrow \infty$. Thus, the axiom says that if $z^{s} \succ x$ for all $s$ sufficiently large, then the pointwise limit of $z^{s}$ (namely, $\left.y\right) \succ x$.

\subsection{The Utilitarian SWR versus the Overtaking SWRs}

We find the ranking of utility streams according to the utilitarian SWR to be persuasive. Consider a situation in which faced with a choice between $x$ and $y$, one finds that there is some $N^{\prime} \in \mathbb{N}$, such that:

$$
\left(I\left(x\left(N^{\prime}\right)\right), x\left[N^{\prime}\right]\right)>\left(I\left(y\left(N^{\prime}\right), y\left[N^{\prime}\right]\right)\right.
$$

Then one can find $N \geq N^{\prime}$ such that:

$$
I(x(N))>I(y(N)) \text { and } x[N] \geq y[N]
$$

Thus, one may consider getting together the members of the finite society $\{1, \ldots, N\}$, and asking them to rank $x$ versus $y$. If they apply utilitarian principles to themselves, they will rank $x$ above $y$. In this case, it is legitimate for the infinite horizon society to rank $x$ above $y$ because the infinite number of future generations, who are not included in the finite society $\{1, \ldots, N\}$, are either indifferent between $x$ and $y$ or prefer $x$ to $y$. In other words, in this situation, all future generations beyond $N$ are willing to go along with the (utilitarian) preferences of the finite society $\{1, \ldots, N\}$.

No such consensus is to be obtained with the overtaking SWR. Consider the following example of two utility streams, where the overtaking SWR 
can compare the two streams and the utilitarian SWR declares them noncomparable.

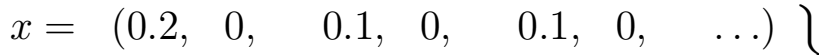

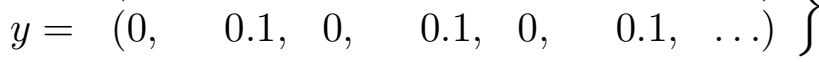

We can verify that for $\bar{N}=1$,

$$
I(x(N))>I(y(N)) \text { for all } N \geq \bar{N}
$$

so that $x \succ y$ according to the overtaking SWR.

The question arises whether $x$ should be preferred to $y$ by the infinite horizon society. This is not altogether clear. The problem with judging $x \succ y$ in such a case can be seen as follows. If we look at any finite-horizon society, and ask the society to rank $x$ versus $y$, they will indeed rank $x$ higher than $y$, if they apply utilitarian principles to themselves. However, no matter how large the finite horizon, there are always an infinite number of future generations who rank $x$ below $y$. Thus, it is never possible to have consensus of opinion between any finite horizon society and the infinite number of future generations not included in that finite society.

The catching up SWR has the further problem that it, in fact, violates the attractive idea of equal treatment that lies behind utilitarianism. Consider a variant of the above example in which:

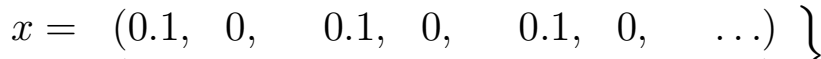

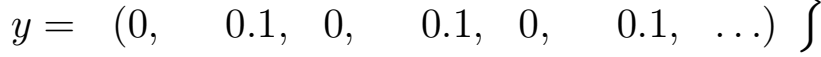

The utilitarian SWR declares $x$ and $y$ non-comparable, and so does the overtaking SWR. However, the catching up SWR declares $x$ preferred to $y$. Note that if the generations $\{1,2,3,4,5,6, \ldots\}$ were rearranged pairwise (this is of course an infinite permuation of $\mathbb{N}$, but of a particularly appealing type) to get $\{2,1,4,3,6,5, \ldots\}$ then the utilities in $x$ would look exactly as in $y$, and vice versa. Since it seems reasonable to have preferences invariant to such rearrangements, it looks like a violation of a basic idea of equal treatment of generations to declare $x$ superior to $y .{ }^{24}$

A comparison of the utilitarian SWR with the overtaking or catchingup SWR can also be made from a somewhat different perspective. Recall

\footnotetext{
${ }^{24}$ Liedekerke and Lauwers (1977, p.162) have also argued that, in this example, $x$ should be judged indifferent to $y$.
} 
that the need to construct such SWRs arises because social welfare functions satisfying Pareto and Anonymity axioms do not exist (and no social welfare order satsfying the two axioms has been constructed). If one discounted future utilities one could get a social welfare function (in our setting) by simply summing up the discounted stream of utilities. The Pareto Axiom would be satisfied but the Anonymity axiom would, of course, be violated. Loosely speaking, this violation could be considered to be "small" for discount factors close to 1 . Thus, one way of checking for robustness of a SWR, satisfying Anonymity and the Pareto axioms, would be to see whether the ranking between two alternatives $x$ and $y$ provided by the SWR is preserved for discount factors close to 1 in the discounted present value SWF.

Note that if $x$ is preferred to $y$ according to the utilitarian SWR, then there is some $N \in \mathbb{N}$, such that $I(x(N))>I(y(N))$ and $x[N] \geq y[N]$. Thus, there is a discount factor $\hat{\delta} \in(0,1)$, such that for all $\delta \in(\hat{\delta}, 1)$, we would have:

$$
\sum_{n=1}^{N} \delta^{n-1} x(n)>\sum_{n=1}^{N} \delta^{n-1} y(n) \text { and } \delta^{n-1} x(n) \geq \delta^{n-1} y(n) \text { for } n \geq N+1
$$

Consequently, for all $\delta \in(\hat{\delta}, 1)$, we would have $f(x ; \delta)>f(y ; \delta)$, where $f(\cdot, \delta)$ is the discounted present value SWF, corresponding to the discount factor $\delta$.

The overtaking SWR does not have this robustness property, and we show this by presenting a concrete example of two utility streams $x$ and $y$, such that $x$ is preferred to $y$ according to the overtaking SWR, but $y$ is preferred to $x$ according to the discounted present value SWF for every $\delta \in(0,1){ }^{25}$

Define $x$ and $y$ as follows:

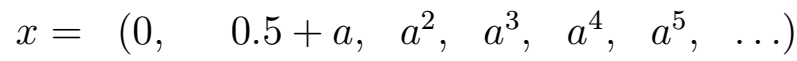

$$
\begin{aligned}
& y=\left(0.5, \quad 0, \quad a, \quad a^{2}, a^{3}, a^{4}, \ldots\right)
\end{aligned}
$$

where $a=(1 / 8)$. Denoting $I(y(N))-I(x(N))$ by $A_{N}$, we see that:

$$
A_{1}=0.5, A_{N}=-a^{N-1} \text { for all } N \geq 2
$$

Clearly then we have:

$$
I(x(N))>I(y(N)) \text { for all } N \geq 2
$$

\footnotetext{
${ }^{25}$ Although the overtaking criterion has been discussed at length in the literature, we are not aware of any paper which presents such an example.
} 
and consequently $x \succ_{O} y$.

We now claim that for the above example, for all $\delta \in(0,1)$,

$$
f(x, \delta) \equiv \sum_{n=1}^{\infty} \delta^{n-1} x(n)<\sum_{n=1}^{\infty} \delta^{n-1} y(n) \equiv f(y, \delta)
$$

Suppose, on the contrary, there is some $\delta \in(0,1)$, such that:

$$
f(x, \delta) \geq f(y, \delta)
$$

Given this $\delta \in(0,1)$, denote $(1-\delta) / 2$ by $\beta$; then $\beta>0$. We can choose $\bar{N} \in \mathbb{N}$ large enough so that:

$$
\delta^{\bar{N}} /(1-\delta) \leq(\beta / 2)
$$

Using (28), note that for all $N \geq \bar{N}$, we have:

$$
\sum_{n=N+1}^{\infty} \delta^{n-1} x(n) \leq \sum_{n=N+1}^{\infty} \delta^{n-1}=\delta^{N} /(1-\delta) \leq \delta^{\bar{N}} /(1-\delta) \leq(\beta / 2)
$$

Using (27) and (29), note that for all $N \geq \bar{N}$, we have:

$$
\begin{aligned}
\sum_{n=1}^{N} \delta^{n-1} x(n) & =\sum_{n=1}^{\infty} \delta^{n-1} x(n)-\sum_{n=N+1}^{\infty} \delta^{n-1} x(n) \\
& =f(x, \delta)-\sum_{n=N+1}^{\infty} \delta^{n-1} x(n) \\
& \geq f(y, \delta)-(\beta / 2) \\
& \geq \sum_{n=1}^{N} \delta^{n-1} y(n)-(\beta / 2)
\end{aligned}
$$

Thus, for all $N \geq \bar{N}$, we obtain:

$$
\sum_{n=1}^{N} \delta^{n-1} y(n)-\sum_{n=1}^{N} \delta^{n-1} x(n) \leq(\beta / 2)
$$


Using (25), we now write for all $N \in \mathbb{N}$,

$$
\begin{aligned}
\sum_{n=1}^{N} \delta^{n-1}(y(n)-x(n))= & A_{1}+\left(A_{2}-A_{1}\right) \delta+\cdots+\left(A_{N}-A_{N-1}\right) \delta^{N-1} \\
= & A_{1}(1-\delta)+\cdots+A_{N-1} \delta^{N-2}(1-\delta)+A_{N} \delta^{N-1} \\
= & 0.5(1-\delta)-(1-\delta)\left[\delta a+\delta^{2} a^{2}+\cdots\right. \\
& \left.+\delta^{N-2} a^{N-2}\right]-\delta^{N-1} a^{N-1} \\
\geq & 0.5(1-\delta)-(1-\delta) \sum_{n=1}^{\infty} \delta^{n} a^{n}-\delta^{N-1} a^{N-1} \\
= & 0.5(1-\delta)-(1-\delta)[\delta a /(1-\delta a)] \\
& -\delta^{N-1} a^{N-1}
\end{aligned}
$$

We have:

$$
[\delta a /(1-\delta a)] \leq[a /(1-a)]=(1 / 7)
$$

and using this information in (31), we obtain for all $N \in \mathbb{N}$,

$$
\sum_{n=1}^{N} \delta^{n-1}(y(n)-x(n)) \geq(5 / 14)(1-\delta)-\delta^{N-1} a^{N-1}
$$

Combining (30) and (32), we obtain for all $N \geq \bar{N}$,

$$
(1-\delta) / 4=(\beta / 2) \geq(5 / 14)(1-\delta)-\delta^{N-1} a^{N-1}
$$

This means that for all $N \geq \bar{N}$, we have:

$$
\delta^{N-1} a^{N-1} \geq(3 / 28)(1-\delta)
$$

Note that the right-hand side of (33) is a positive constant (since $\delta \in(0,1)$ is given) independent of $N$. The left-hand side of (33) depends on $N$, and goes to zero as $N \rightarrow \infty$. This contradiction establishes our claim (26). That is $y$ is preferred to $x$ according to the present discounted value SWF for every $\delta \in(0,1)$.

Finally, we note that the utilitarian SWR, despite being more incomplete than the overtaking SWR and the catching up SWR, provides sufficient comparability to study "optimal" behavior in many intertemporal allocation models. For instance, in a model of forestry management, Mitra (2003) has 
shown that the nature of the production process implies that the notion of optimality according to the catching up SWR and the corresponding notion of maximality according to the utilitarian SWR coincide. Thus, the additional power of comparison, gained by using the overtaking SWR or catching up SWR, is superfluous in this context. 


\section{References}

[1] Asheim, G.B., W.Buchholz and B. Tungodden, Justifying Sustainability, Journal of Environmental Economics and Management 41 (2001), 252268.

[2] Asheim, G.B. and B. Tungodden, Resolving Distributional Conflicts Between Generations, mimeo, 2002.

[3] Atsumi, H.: Neoclassical Growth and the Efficient Program of Capital Accumulation, Review of Economic Studies, 32 (1965), 127-136.

[4] Basu, K.: Cardinal Utility, Utilitarianism and a Class of Invariance Axioms in Welfare Analysis, Journal of Mathematical Economics 12 (1983).

[5] Basu, K. and T. Mitra: Aggregating Infinite Utility Streams with Intergenerational Equity: the Impossibility of Being Paretian, (2002), forthcoming in Econometrica.

[6] Blackorby, C., D. Donaldson and J.A. Weymark: Social Choice with Interpersonal Utility Comparisons: A Diagrammatic Introduction, International Economic Review 25 (1984), 327-356.

[7] Brock, W.A.: An Axiomatic Basis for the Ramsey-Weizsacker Overtaking Criterion, Econometrica 38 (1970), 927-929.

[8] d'Aspremont, C. and L. Gevers: Equity and the Informational Basis of Collective Choice, Review of Economic Studies 46 (1977), 199-210.

[9] Debreu, G.: Topological Methods in Cardinal Utility Theory, in K. J. Arrow, S. Karlin and P. Suppes, Eds., Mathematical Methods in the Social Sciences; Stanford, Stanford University Press, 1960.

[10] Debreu, G.: Theory of Value, New York, John Wiley, 1959.

[11] Diamond, P.: The Evaluation of Infinite Utility Streams, Econometrica 33 (1965), 170-177.

[12] Fishburn, P.C.: Utility Theory for Decision Making; New York, John Wiley, 1970. 
[13] Koopmans, T.C.: Stationary Ordinal Utility and Impatience, Econometrica 28 (1960), 287-309.

[14] Koopmans, T.C., P.A. Diamond, and R.E. Williamson: Stationary Utility and Time Perspective, Econometrica 32 (1964), 82-100.

[15] Lauwers, L.: Rawlsian Equity and Generalized Utilitarianism with an Infinite Population, Economic Theory 9 (1997), 143-150.

[16] Liedekerke, L. and L. Lauwers: Sacrificing the Patrol:Utilitarianism, Future Generations and Infinity, Economics and Philosophy 13 (1997), 159-174.

[17] Maskin, E.: A Theorem on Utilitarianism, Review of Economic Studies 45 (1978), 93-96.

[18] Milnor, J.: Games Against Nature, Chapter IV in R.M. Thrall, C.H. Coombs, R.L. Davis, Eds., Decision Processes; New York, John Wiley, 1954.

[19] Mitra, T.: Intergenerational Equity and the Forest Management Problem, mimeo, Cornell University, 2003.

[20] Roberts, K.W.S.: Interpersonal Comparability and Social Choice Theory, Review of Economic Studies 47 (1980), 421-39.

[21] Sen, A.K.: Collective Choice and Social Welfare; Edinburgh, Oliver\&Boyd, 1971.

[22] Sen, A.K.: On Weights and Measures: Informational Constraints in Social Welfare Analysis, Econometrica 45 (1977), 1539-72.

[23] Suppes, P.: Some Formal Models of Grading Principles, Synthese 6 (1966), 284-306.

[24] Svensson, L.-G.: Equity among Generations, Econometrica 48 (1980), 1251-1256.

[25] von Weizsäcker, C. C.: Existence of Optimal Programs of Accumulation for an Infinite Time Horizon, Review of Economic Studies, 32 (1965), 85-104. 